\title{
Scaling relations for galaxy clusters
}

\author{
C. Marmo ${ }^{1}$, G. Fasano ${ }^{2}$, E. Pignatelli ${ }^{2}$, B. Poggianti ${ }^{2}$, \\ D. Bettoni ${ }^{2}$, C. Halliday ${ }^{2}$, J. Varela ${ }^{3}$, M. Moles ${ }^{4}$, \\ P. Kjærgaard ${ }^{5}$, W. Couch $^{6}$ and A. Dressler ${ }^{7}$ \\ ${ }^{1}$ Astronomy Department, University of Padova, Italy email: marmo@pd.astro.it \\ ${ }^{2}$ INAF, Osservatorio Astronomico di Padova, Italy \\ ${ }^{3}$ IMAFF, CSIC, Madrid, Spain \\ ${ }^{4}$ Instituto de Astrofísica de Andalucía, Granada, Spain \\ ${ }^{5}$ Copenhagen University Observatory, Copenhagen, Denmark \\ ${ }^{6}$ School of Physics, University of New South Wales, Sydney, Australia \\ ${ }^{7}$ Observatories of the Carnegie Institution of Washington, Pasadena, USA
}

\begin{abstract}
We use preliminary results of the WINGS survey (Fasano et al.) to obtain determinations of optical scaling relations for galaxy clusters. Passing from one- to two- parameter scaling relations we pay particular attention to the Kormendy relation (KR) and to the Fundamental Plane (FP) of galaxy clusters, comparing them with scaling relations of elliptical galaxies.
\end{abstract}

\section{Cluster profiles}

Spherical symmetry has been assumed in determining the cluster surface brightness profile. Then, galaxies have been counted in a series of a concentric rings and integrated magnitudes have been obtained multiplying each galaxy by its own light in the $\mathrm{V}$ band. We correct the $\mathrm{V}$ magnitudes for galactic extinction, according to Burstein \& Heiles (1982). The correction for back and foreground is made statistically, referring to the galaxy counts of Cabanac et al. (2000). Due to the dimension and shape of the wide-field detectors we had to apply an area correction, in order to circularize the rectangular limits of the field. We counted galaxies in each ring down to an apparent $\mathrm{V}$ magnitude of 20.5 mag. Then, we corrected for luminosity function incompletness. This correction turned out to be of the order of $1 \%$ of the total luminosity. Finally we fit the growth curve of the luminosity profile with a de Vaucouleurs law.

\section{One-parameter scaling relations}

While King and, in general, cored profiles reproduce the galactic luminosity profile of clusters better than the de Vaucouleurs one, we use the luminosity extrapolated from the de Vaucouleurs profile to make a comparison with ellipticals. Moreover, different definitions of luminosity can strongly affect the determination of some scaling relations.

In Figure 1 the optical luminosities of our clusters are plotted versus X-Ray luminosities and velocity dispersions from the literature. In particular, the top panels refer to the total luminosities derived from de Vaucouleurs fits of the profiles, respectively. Instead, the bottom panels report the luminosities obtained from the galaxy counts in our catalogues, just correcting for background contamination and for incompleteness of the area $\left(L_{\text {Acorr }}\right)$. The solid lines in the plots represent our linear, weighted best fits to the data. It is evident that the slopes and r.m.s. of our fits decrease from the top to the bottom panels. On the other hand, the quantity $L_{A c o r r}$, rather than of the total cluster light, 

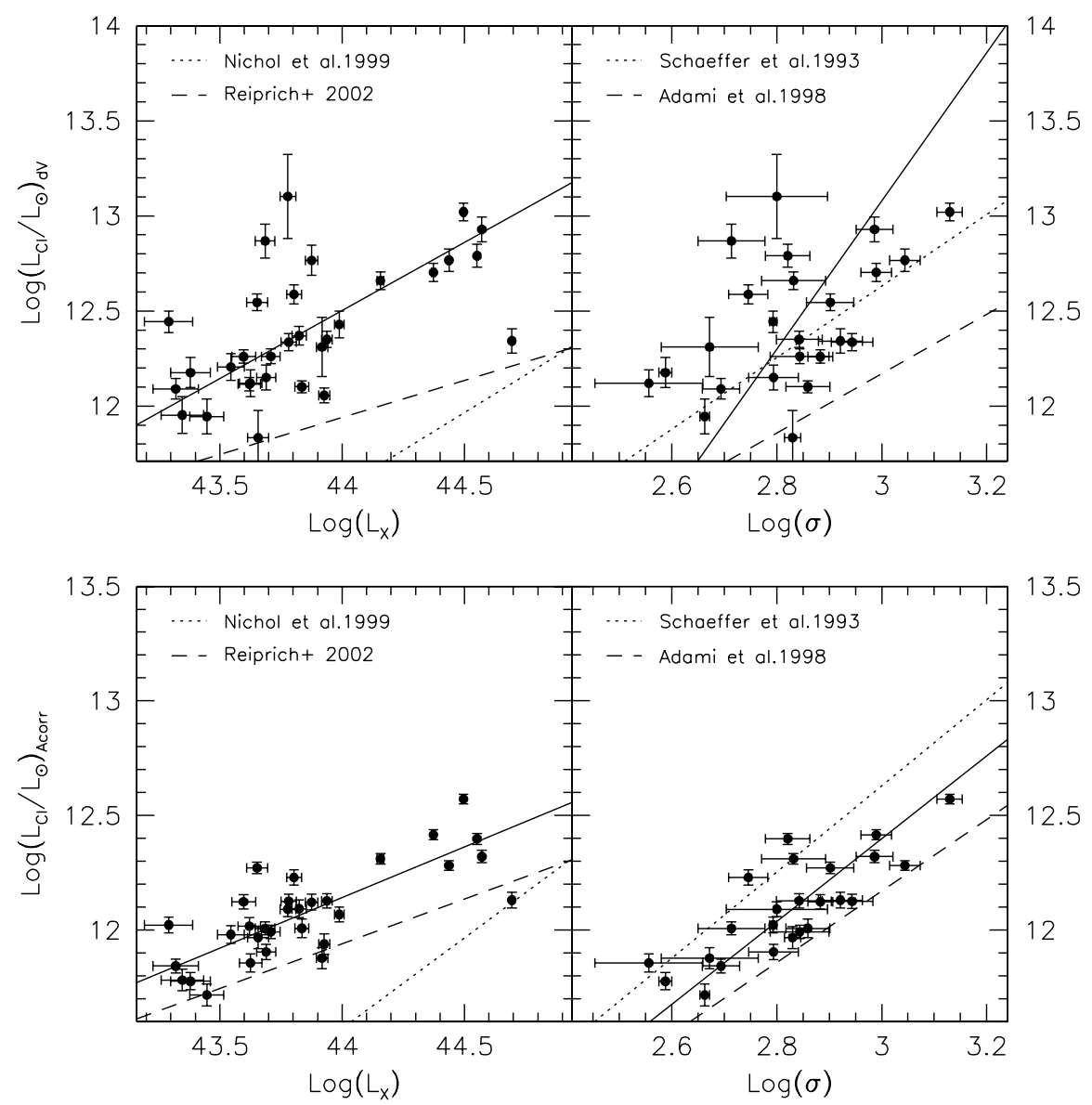

Figure 1. Left panels: total V luminosity versus X-ray luminosity: Nichol et al. (1999) (dotted lines) and Reiprich \& Böringer (2002) (dashed lines). Right panels: total V luminosity versus velocity dispersion: dotted lines represent $L_{V}-\sigma$ fit from Schaeffer et al. (1993), dashed lines that from Adami et al. (1998).

is likely indicative of the light coming from some inner cluster region, presumably (and roughly) corresponding to the dynamically/structurally relaxed core. This would explain why it shows better correlations with dynamical and mass-density indicators, like $\sigma$ and $L_{X}$.

\section{The Kormendy relation for galaxy clusters}

In spite of the fact that stars (in elliptical galaxies) and galaxies (in clusters) experience quite different dynamical regimes, the impressive similarity among KR relative to systems having so much different scales (see Figure 2) is likely to reflect a unified density-radius relation for luminous matter. The large scatter shown by galaxies on the $\left\langle\mu_{e}\right\rangle-R_{e}$ plane could possibly reflect a more complex dynamical and structural evolution of galaxies with respect to clusters. These aspects of the extended Kormendy relation will require a careful analysis and an exaustive discussion. 

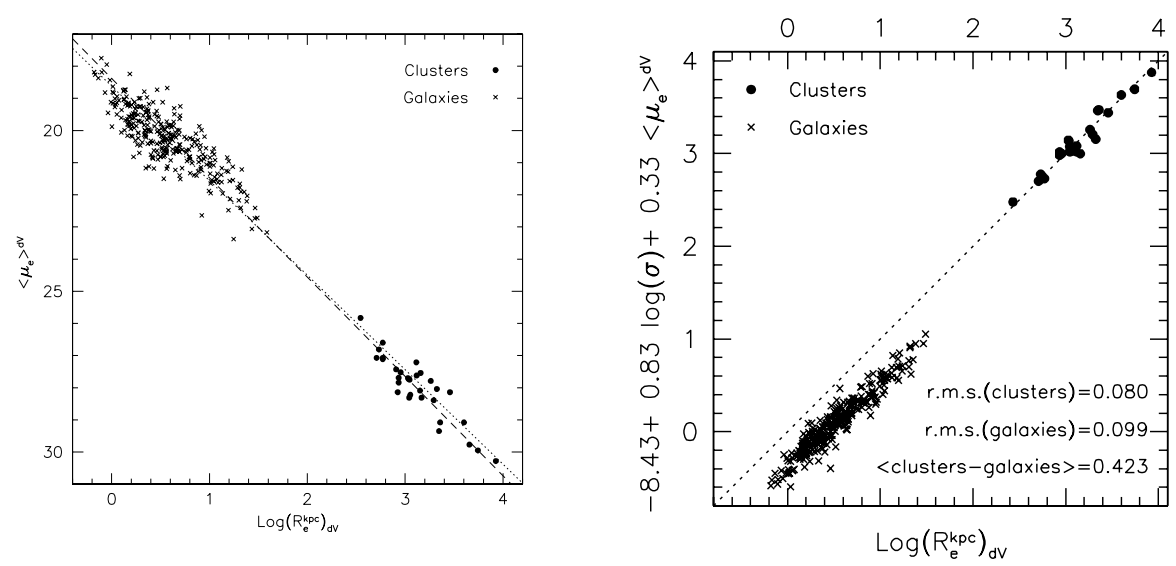

Figure 2. The Kormendy relation (left) and the Fundamental Plane (right) for elliptical galaxies and for clusters. Data for elliptical galaxies are taken from Bettoni et al. (2001).

\section{The Fundamental Plane for galaxy clusters}

By analogy with the 'extended Kormendy relation', we use our sample of clusters to illustrate in Figure 2 an 'extended FP' of gravitationally bound systems. This was first suggested by Schaeffer et al. (1993) with the aim to combine, in the same parameter space, systems spanning very different scales of mass and radii. Again, the slope of the $\mathrm{FP}$ of clusters is very close to that of galaxies, but in this case the zero points are very different, due to the fact that the mass-to-light ratio of clusters is about two orders of magnitudes greater than that of galaxies.

\section{References}

Adami, C., Mazure, A., Biviano, A., Katgert, P., Rhee, G. A\&A 1998, 331, 493.

Bettoni, D., Falomo, R., Fasano, G., Govoni, F., Salvo, M., Scarpa, R. A\&A 2001, 380, 471.

Burstein, D., Heiles, C. AJ 1982, 87, 1165.

Cabanac, R. A., de Lapparent, V., Hicson, P. A\& A 2000, 364, 349.

Fasano, G., Bettoni, D., Marmo, C., Pignatelli, E., Poggianti, B. M., Moles, M., Kjærgaard, P. ASP Conf. 2002, 268, 361.

Nichol, R. C., Melott, A., Miller, C. J. AAS 1999, 195, 1001.

Reiprich, T. H., Böringer, H., ApJ 2002, 567, 716.

Schaeffer, R., Maurogordato, S., Cappi, A., Bernardeau, F. MNRAS 1993, 301, 21L. 